\title{
On existence results for solutions of a coupled system of hybrid boundary value problems with hybrid conditions
}

\author{
Dumitru Baleanu ${ }^{1,2^{*}}$, Hasib Khan ${ }^{3,4}$, Hossein Jafari ${ }^{5,6}$, Rahmat Ali Khan ${ }^{3}$ and Mohsen Alipour ${ }^{7}$
}

\author{
"Correspondence: \\ dumitru@cankaya.edu.tr \\ ${ }^{1}$ Department of Mathematics and \\ Computer Science, Cankaya \\ University, Ankara, 06530, Turkey \\ ${ }^{2}$ Institute of Space Sciences, P.O. Box \\ MG-23, Magurele-Bucharest, 76900, \\ Romania \\ Full list of author information is \\ available at the end of the article
}

\begin{abstract}
We investigate sufficient conditions for existence and uniqueness of solutions for a coupled system of fractional order hybrid differential equations (HDEs) with multi-point hybrid boundary conditions given by

$$
\begin{aligned}
& \mathcal{D}^{\omega}\left(\frac{x(t)}{\mathcal{H}(t, x(t), z(t))}\right)=-\mathcal{K}_{1}(t, x(t), z(t)), \quad \omega \in(2,3] \\
& \mathcal{D}^{\epsilon}\left(\frac{z(t)}{\mathcal{G}(t, x(t), z(t))}\right)=-\mathcal{K}_{2}(t, x(t), z(t)), \quad \epsilon \in(2,3] \\
& \left.\frac{x(t)}{\mathcal{H}(t, x(t), z(t))}\right|_{t=1}=0,\left.\quad \mathcal{D}^{\mu}\left(\frac{x(t)}{\mathcal{H}(t, x(t), z(t))}\right)\right|_{t=\delta_{1}}=0, \quad x^{(2)}(0)=0, \\
& \left.\frac{z(t)}{\mathcal{G}(t, x(t), z(t))}\right|_{t=1}=0,\left.\quad \mathcal{D}^{v}\left(\frac{z(t)}{\mathcal{G}(t, x(t), z(t))}\right)\right|_{t=\delta_{2}}=0, \quad z^{(2)}(0)=0,
\end{aligned}
$$

where $t \in[0,1], \delta_{1}, \delta_{2}, \mu, v \in(0,1)$, and $\mathcal{D}^{\omega}, \mathcal{D}^{\epsilon}, \mathcal{D}^{\mu}$ and $\mathcal{D}^{v}$ are Caputo's fractional derivatives of order $\omega, \epsilon, \mu$ and $\nu$, respectively, $\mathcal{K}_{1}, \mathcal{K}_{2} \in C([0,1] \times \mathcal{R} \times \mathcal{R}, \mathcal{R})$ and $\mathcal{G}, \mathcal{H} \in C([0,1] \times \mathcal{R} \times \mathcal{R}, \mathcal{R}-\{0\})$. We use classical results due to Dhage and Banach's contraction principle (BCP) for the existence and uniqueness of solutions. For applications of our results, we include examples.
\end{abstract}

Keywords: coupled system of hybrid fractional differential equations; existence of solutions; uniqueness of solutions

\section{Introduction}

Scientists working in various disciplines of science are interested in getting better interpretations of their results by incorporating more and more information in their models. For this purpose they are utilizing different mathematical tools in their models, one of them is the use of fractional order derivatives. Fractional order differential equations (FDEs) capture more information as compared to ordinary differential equations and are believed to be a better approach in mathematical modeling of many scientific problems. Recently, FDEs have been frequently used in biology, economics, polymer rheology, chemistry, mechanics, aerodynamics, control theory, regular variation in thermodynamics, biophysics, signal and image processing etc. [1-3].

(c) 2015 Baleanu et al. This article is distributed under the terms of the Creative Commons Attribution 4.0 International License (http://creativecommons.org/licenses/by/4.0/), which permits unrestricted use, distribution, and reproduction in any medium, provided you give appropriate credit to the original author(s) and the source, provide a link to the Creative Commons license, and indicate if changes were made. 
Recently, the theory on existence, uniqueness and multiplicity of nonlinear boundary value problems (BVPs) for FDEs has attracted much attention and is a fast growing area of research. We refer to $[4,5]$ and the references therein for some of valuable and recent achievements in the study of existence theory for BVPs corresponding to FDEs.

Existence and uniqueness of solutions (EUS) for coupled systems of fractional order differential equations (CSFDEs) have also attracted the attention of many scientists, for example, Rehman and Khan [6], Li and Liu [7], Ntouyas and Obaid [8]. Hybrid differential equations were explored by Hedrih [9] and Dhage [10,11]. Ahmad et al. [12] investigated a coupled system of hybrid fractional order differential equations (CSHFDEs) by the help of Banach's fixed point theorem and Leray-Schauder's alternative, they considered the fractional derivative in Caputo's sense. Herzallah and Baleanu [13], Ahmad and Ntouyas [14] discussed the existence of solutions for hybrid FDEs with initial value problems by a fixed point theorem due to Dhage [15].

In this paper, we study EUS for the following coupled system of HFDEs:

$$
\begin{aligned}
& \mathcal{D}^{\omega}\left(\frac{x(t)}{\mathcal{H}(t, x(t), z(t))}\right)=-\mathcal{K}_{1}(t, x(t), z(t)), \quad \omega \in(2,3], \\
& \mathcal{D}^{\epsilon}\left(\frac{z(t)}{\mathcal{G}(t, x(t), z(t))}\right)=-\mathcal{K}_{2}(t, x(t), z(t)), \quad \epsilon \in(2,3], \\
& \left.\frac{x(t)}{\mathcal{H}(t, x(t), z(t))}\right|_{t=1}=0,\left.\quad \mathcal{D}^{\mu}\left(\frac{x(t)}{\mathcal{H}(t, x(t), z(t))}\right)\right|_{t=\delta_{1}}=0, \quad x^{(2)}(0)=0, \\
& \left.\frac{z(t)}{\mathcal{G}(t, x(t), z(t))}\right|_{t=1}=0,\left.\quad \mathcal{D}^{v}\left(\frac{z(t)}{\mathcal{G}(t, x(t), z(t))}\right)\right|_{t=\delta_{2}}=0, \quad z^{(2)}(0)=0,
\end{aligned}
$$

where $\delta_{1}, \delta_{2} \in(0,1), \omega, \epsilon \in(2,3], t \in[0,1]$ and $D^{\omega}, D^{\epsilon}, D^{\mu}, D^{\nu}$ are Caputo's fractional derivatives of orders $\omega, \epsilon, \mu, v$, respectively, $\mathcal{K}_{1}, \mathcal{K}_{2} \in C\left([0,1] \times \mathcal{R}^{2}, \mathcal{R}\right), \mathcal{H}, \mathcal{G} \in C([0,1] \times$ $\left.\mathcal{R}^{2}, \mathcal{R}-\{0\}\right)$ and $x, z \in C([0,1], \mathcal{R})$. Here we remark that $\mathcal{H}(t, x, z)$ can not be of $O(x)$ and $\mathcal{G}(t, x(t), z(t))$ can not be of $O(z)$. Because if $\mathcal{H}(t, x, z)$ is of $O(x)$ and $\mathcal{G}(t, x(t), z(t))$ is of $O(z)$, then system (1) will reduce to an algebraic coupled system of equations and will lose its hybrid nature.

It is investigated that dynamics of multi deformable bodies such as beams, plates, membranes etc. coupled by standard light fractional order discrete continuous layers is described by coupled fractional order hybrid system dynamics [9]. These are generally perturbed fractional order differential equations, and they are mainly classified as perturbed differential equations of the first and second type. The perturbation itself is of two types, namely linear and quadratics, whose details can be studied in [9-11]. Here, our system (1) is not an artificial one but in fact a quadratic perturbation of the second type. These types of systems are generally tackled with the use of hybrid fixed point theory. The significance of the hybrid system of differential equations lies in the fact that they include several dynamical systems as special cases. For example, if we choose $\mathcal{H}(t, x, z)=1, \mathcal{G}(t, x, z)=1$ as constant functions, then our system (1) will reduce to a class of coupled system of bound- 
ary value problems for fractional order differential equations of the type:

$$
\begin{aligned}
& \mathcal{D}^{\omega} x(t)=-\mathcal{K}_{1}(t, x(t), z(t)), \quad \omega \in(2,3], \\
& \mathcal{D}^{\epsilon} z(t)=-\mathcal{K}_{2}(t, x(t), z(t)), \quad \epsilon \in(2,3], \\
& \left.x(t)\right|_{t=1}=0,\left.\quad \mathcal{D}^{\mu} x(t)\right|_{t=\delta_{1}}=0, \quad x^{(2)}(0)=0, \\
& \left.z(t)\right|_{t=1}=0,\left.\quad \mathcal{D}^{v} z(t)\right|_{t=\delta_{2}}=0, \quad z^{(2)}(0)=0 .
\end{aligned}
$$

Organization of the paper: In this section, we give the basic and related work to the existence of solutions of FDEs by the use of some classical results; also the auxiliary lemma of this paper is presented, and some important work which has stimulated us for this study is cited. In Section 2, we give the main two lemmas of the paper in two subsections for the existence of solutions (ES) and uniqueness of solutions (US) for the coupled system of HFDEs (1). In the final section, we give two illustrative examples for demonstration of our results, which affirm the EUS for CSHFDEs (1).

We recall the following definitions of fractional order integral, fractional order derivative in Caputo's sense and some basic results of fractional calculus [2, 3].

Definition 1 [13] If $f(t) \in L^{1}(a, b)$, the set of all integrable functions, and $\omega>0$, then the left Riemann-Liouville fractional integral of order $\omega$ is defined by

$$
I^{\omega} f(t)=\frac{1}{\Gamma(\omega)} \int_{a}^{t}(t-x)^{\omega-1} f(x) d x
$$

Definition 2 [13] For $\omega>0$, the left Caputo fractional derivative of order $\omega$ is defined by

$$
\mathcal{D}^{\omega} f(t)=\frac{1}{\Gamma(n-\omega)} \int_{0}^{t}(t-x)^{n-\omega-1} \mathcal{D}^{n} f(x) d x,
$$

where $n$ is such that $n-1<\omega<n$ and $\mathcal{D}=\frac{d}{d x}$.

Lemma 3 [2] For $\omega, \beta>0$, the following relation holds:

$$
\mathcal{D}^{\omega} t^{\epsilon}=\frac{\Gamma(1+\epsilon)}{\Gamma(1+\epsilon-\omega)} t^{\epsilon-\omega-1}, \quad \epsilon>n, \quad D^{\omega} t^{k}=0, \quad k=0,1, \ldots, n-1 .
$$

Lemma 4 [3] Let $a, b \geq 0$ and $f \in L_{1}[p, q]$. Then $I_{0^{+}}^{a} I_{0^{+}}^{b} f(t)=I_{0^{+}}^{a+b} f(t)=I_{0^{+}}^{b} a_{0^{+}}^{a} f(t)$ and ${ }^{c} D_{0^{+}}^{b} I_{0^{+}}^{b} f(t)=f(t)$ for all $t \in[p, q]$.

Lemma 5 [2] For $\epsilon \geq \omega>0$ and $f(t) \in L_{1}[a, b]$, the following holds:

$$
D^{\omega} I_{a+}^{\epsilon} f(t)=I_{a+}^{\epsilon-\omega} f(t)
$$

on the interval $[a, b]$ iff $f \in C[a, b]$.

Lemma 6 [2] For $k-1<\omega \leq k$ and $f(t) \in A C^{k}(0,1)$, the solution of homogenous FDE $D_{0^{+}}^{\omega} f(t)=0$ is

$$
f(t)=c_{1}+c_{2} t+c_{3} t^{2}+\cdots+c_{k} t^{k-1}, \quad k_{i} \in R, i=1,2,3, \ldots, k .
$$


We present the following lemma as the auxiliary lemma of this paper for the EUS for CSHFDEs (1).

Lemma 7 For $x \in A C^{3}[0,1]$ and any $\mathcal{Z} \in C[0,1]$, a solution of the $H B V P$

$$
\begin{aligned}
& \mathcal{D}^{\omega}\left(\frac{x(t)}{\mathcal{H}(t, x, z)}\right)=-\mathcal{Z}(t), \quad \omega \in(2,3], \\
& \left.\frac{x(t)}{\mathcal{H}(t, x, z)}\right|_{t=1}=0,\left.\quad \mathcal{D}^{\mu}\left(\frac{x(t)}{\mathcal{H}(t, x, z)}\right)\right|_{t=\delta_{1}}=0, \quad x^{(2)}(0)=0, \quad \mu \in(0,1),
\end{aligned}
$$

is given by

$$
\begin{aligned}
x(t)= & \mathcal{H}(t, x, z)\left[-\int_{0}^{t} \frac{(t-s)^{\omega-1}}{\Gamma(\omega)} \mathcal{Z}(s) d s+\int_{0}^{1} \frac{(1-s)^{\omega-1}}{\Gamma(\omega)} \mathcal{Z}(s) d s\right. \\
& \left.+\frac{(t-1)}{\Delta_{1} \Gamma(\omega-\mu)} \int_{0}^{\delta_{1}}\left(\delta_{1}-s\right)^{\omega-\mu-1} \mathcal{Z}(s) d s\right] .
\end{aligned}
$$

Proof Applying the operator $I^{\omega}$ on the differential equation in (7), we obtain

$$
\frac{x(t)}{\mathcal{H}(t, x, z)}=-I^{\omega} \mathcal{Z}(t)+c_{1}+c_{2} t+c_{3} t^{2}
$$

By the use of initial condition $x^{(2)}(0)=0$, we get $c_{3}=0$, and hence (9) takes the form

$$
\frac{x(t)}{\mathcal{H}(t, x, z)}=-I^{\omega} \mathcal{Z}(t)+c_{1}+c_{2} t
$$

Applying Caputo's fractional derivative of order $\mu$ to (10), we get

$$
\mathcal{D}^{\mu}\left(\frac{x(t)}{\mathcal{H}(t, x, z)}\right)=-I^{\omega-\mu} \mathcal{Z}(t)+c_{2} \frac{t^{1-\mu}}{\Gamma(2-\mu)} .
$$

The boundary condition $\left.\mathcal{D}^{\mu}\left(\frac{x(t)}{\mathcal{H}(t, x, \Omega)}\right)\right|_{t=\delta_{1}}=0$ yields $c_{2}=\frac{I^{\omega-\mu}}{\Delta_{1}} \mathcal{Z}\left(\delta_{1}\right)$, where $\Delta_{1}=\frac{\delta_{1}^{1-\mu}}{\Gamma(2-\mu)}$, and the boundary condition $\left.\frac{x}{\mathcal{H}(t, x, x)}\right|_{t=1}=0$ yields $c_{1}=I^{\omega} \mathcal{Z}(1)-\frac{I^{(\omega-\mu}}{\Delta_{1}} \mathcal{Z}\left(\delta_{1}\right)$. Thus, (11) becomes

$$
\frac{x(t)}{\mathcal{H}(t, x, z)}=-I^{\omega} \mathcal{Z}(t)+I^{\omega} \mathcal{Z}(1)+(t-1) \frac{I^{\omega-\mu}}{\Delta_{1}} \mathcal{Z}\left(\delta_{1}\right)
$$

which implies that

$$
\begin{aligned}
x(t)= & \mathcal{H}(t, x, z)\left[-\int_{0}^{t} \frac{(t-s)^{\omega-1}}{\Gamma(\omega)} \mathcal{Z}(s) d s+\int_{0}^{1} \frac{(1-s)^{\omega-1}}{\Gamma(\omega)} \mathcal{Z}(s) d s\right. \\
& \left.+\frac{(t-1)}{\Delta_{1} \Gamma(\omega-\mu)} \int_{0}^{\delta_{1}}\left(\delta_{1}-s\right)^{\omega-\mu-1} \mathcal{Z}(s) d s\right] .
\end{aligned}
$$

Thus, the proof is completed. 


\section{Main results}

In this section, we are concerned with the EUS for CSHFDEs (1) by using some classical results. For this, consider the Banach space $X=\{x(t): x(t) \in C[0,1]\}$ with norm $\|x\|=$ $\max _{t \in[0,1]}|x(t)|$ and $(X \times X,\|(\cdot, \cdot)\|)$ with norm $\|(x, z)\|=\|x\|+\|z\|$. We define an operator $\mathcal{F}: X \times X \rightarrow X \times X$ by

$$
\mathcal{F}(x, z)(t)=\left(\mathcal{F}_{1}(x, z)(t), \mathcal{F}_{2}(x, z)(t)\right)
$$

where

$$
\begin{aligned}
\mathcal{F}_{1}(x, z)(t)= & \mathcal{H}(t, x, z)\left[-\int_{0}^{t} \frac{(t-s)^{\omega-1}}{\Gamma(\omega)} \mathcal{K}_{1}(s, x(s), z(s)) d s\right. \\
& +\int_{0}^{1} \frac{(1-s)^{\omega-1}}{\Gamma(\omega)} \mathcal{K}_{1}(s, x(s), z(s)) d s \\
& \left.+\frac{(t-1)}{\Delta_{1} \Gamma(\omega-\mu)} \int_{0}^{\delta_{1}}\left(\delta_{1}-s\right)^{\omega-\mu-1} \mathcal{K}_{1}(s, x(s), z(s)) d s\right]
\end{aligned}
$$

and

$$
\begin{aligned}
\mathcal{F}_{2}(x, z)(t)= & \mathcal{G}(t, x, z)\left[-\int_{0}^{t} \frac{(t-s)^{\epsilon-1}}{\Gamma(\epsilon)} \mathcal{K}_{2}(s, x(s), z(s)) d s\right. \\
& +\int_{0}^{1} \frac{(1-s)^{\epsilon-1}}{\Gamma(\epsilon)} \mathcal{K}_{2}(s, x(s), z(s)) d s \\
& \left.+\frac{(t-1)}{\Delta_{2} \Gamma(\epsilon-v)} \int_{0}^{\delta_{2}}\left(\delta_{2}-s\right)^{\epsilon-\nu-1} \mathcal{K}_{2}(s, x(s), z(s)) d s\right],
\end{aligned}
$$

where $\Delta_{1}=\frac{\delta_{1}^{1-\mu}}{\Gamma(2-\mu)}, \Delta_{2}=\frac{\delta_{2}^{1-\nu}}{\Gamma(2-\nu)}$. In view of Lemma 7, the solutions of CSHFDEs (1) are fixed points of the operator $\mathcal{F}$. Define the following terms:

$$
\begin{aligned}
& \mathcal{N}_{1}=\frac{2}{\Gamma(\omega+1)}+\frac{2 \delta_{1}^{\omega-\mu}}{\Delta_{1} \Gamma(\omega-\mu+1)} \\
& \mathcal{N}_{2}=\frac{2}{\Gamma(\epsilon+1)}+\frac{2 \delta_{2}^{\epsilon-\nu}}{\Delta_{2} \Gamma(\epsilon-v+1)}
\end{aligned}
$$

\subsection{Existence of solutions}

In this subsection, we are interested in the existence of solutions of CSHFDEs (1). For this purpose, we take help from the following fixed point theorem.

Theorem $8[13,15]$ Let $\mathcal{X}_{a}(0)$ and $\overline{\mathcal{X}_{a}(0)}$ be open and closed balls in a Banach algebra $X$ centered at the origin 0 of radius a for some real number $a$, and let $\mathcal{A}, \mathcal{B}: \overline{\mathcal{X}_{a}(0)} \rightarrow X$ be two operators satisfying the following:

(1) $\mathcal{A}$ is Lipschitz with Lipschitz constant $\lambda$;

(2) $\mathcal{B}$ is continuous and compact;

(3) $\lambda \mathcal{M}<1$, where $\mathcal{M}=\| \mathcal{B}\left(\overline{\left.\mathcal{X}_{a}(0)\right)} \|=\sup \left\{\|\mathcal{B}(x)\|: x \in \overline{\mathcal{X}_{a}(0)}\right\}\right.$.

Then, either

(a) the equation $\mathcal{A}(x) \mathcal{B}(x)=x$ has a solution in $\overline{\mathcal{X}_{a}(0)}$, or

(b) there is an element $x \in X$ such that $\|x\|=a$ satisfying $\mu \mathcal{A} x \mathcal{B} x=x$ for some $0<\mu<1$. 
Definition 9 [13] Let $X$ be a Banach space. A mapping $\mathcal{F}: X \rightarrow X$ is called $\mathcal{D}$-Lipschitzian if there exists a continuous and nondecreasing function $\psi: R \rightarrow R$ such that $\|\mathcal{F} x-\mathcal{F} y\| \leq$ $\psi\|x-y\|$ for all $x, y \in X$, where $\psi(0)=0$. If $\psi$ is not necessarily nondecreasing and satisfies $\psi(a)<a$ for $a>0$, the mapping $\mathcal{F}$ is called a nonlinear contraction with a contraction function $\psi$.

\section{Theorem 10 Assume the following:}

$\left(C_{1}\right)$ There exist real constants $\mu_{i}>0($ for $i=1,2,3,4)$ such that $\left|\mathcal{H}\left(t, x_{1}, z_{1}\right)-\mathcal{H}\left(t, x_{2}, z_{2}\right)\right| \leq$ $\mu_{1}\left|x_{1}-x_{2}\right|+\mu_{2}\left|z_{1}-z_{2}\right|,\left|\mathcal{G}\left(t, x_{1}, z_{1}\right)-\mathcal{G}\left(t, x_{2}, z_{2}\right)\right| \leq \mu_{3}\left|x_{1}-x_{2}\right|+\mu_{4}\left|z_{1}-z_{2}\right|$, for $\left(t, x_{j}, z_{j}\right) \in$ $([0,1] \times \mathcal{R} \times \mathcal{R}), j=1,2$.

$\left(C_{2}\right)$ There exist functions $\mathcal{M}_{i}(t) \in C[0,1]$ (for $\left.i=1,2\right)$ such that $\left|\mathcal{K}_{1}(t, x, z)\right| \leq \mathcal{M}_{1}(t)$, $\left|\mathcal{K}_{2}(t, x, z)\right| \leq \mathcal{M}_{2}(t)$ for $(t, x, z) \in([0,1] \times \mathcal{R} \times \mathcal{R})$.

(C) $\gamma\left(\mathcal{N}_{1}\left\|\mathcal{M}_{1}\right\|+\mathcal{N}_{2}\left\|\mathcal{M}_{2}\right\|\right)<1$, for $\gamma=\mu_{1}+\mu_{2}+\mu_{3}+\mu_{4}$.

(C) $\frac{\lambda}{1-\rho}\left(\frac{\mathcal{H}_{0} \mathcal{N}_{1}\left\|\mathcal{M}_{1}\right\|}{1-\lambda \mathcal{N}_{1}\left\|\mathcal{M}_{1}\right\|}+\frac{\mathcal{G}_{0} \mathcal{N}_{2}\left\|\mathcal{M}_{2}\right\|}{1-\lambda \mathcal{N}_{2}\left\|\cdot \mathcal{M}_{2}\right\|}\right)<$ for $\mathcal{H}_{0}=\max _{t \in[0,1]}|\mathcal{H}(t, 0,0)|$ and $\mathcal{G}_{0}=\max _{t \in[0,1]} \mid \mathcal{G}(t$, $0,0) \mid$ and $\rho=\max \left\{\frac{\lambda \mathcal{N}_{1}\left\|\mathcal{M}_{1}\right\|}{1-\lambda \mathcal{N}_{1}\left\|\mathcal{M}_{1}\right\|}, \frac{\lambda \mathcal{N}_{2}\left\|\mathcal{M}_{2}\right\|}{1-\lambda \mathcal{N}_{2}\left\|\mathcal{M}_{2}\right\|}\right\}$, where $\lambda \in(0,1)$.

Then CSHFDEs (1) has a solution.

Proof We give the proof in the following four steps.

The operators $\mathcal{F}_{1}, \mathcal{F}_{2}$ given in (15), (16) are equivalent to

$$
\begin{array}{ll}
\mathcal{F}_{1}(x(t), z(t))=\mathcal{A}_{1}(x(t), z(t)) \mathcal{B}_{1}(x(t), z(t))=(x(t), z(t)), & t \in[0,1], \\
\mathcal{F}_{2}(x(t), z(t))=\mathcal{A}_{2}(x(t), z(t)) \mathcal{B}_{2}(x(t), z(t))=(x(t), z(t)), & t \in[0,1] .
\end{array}
$$

Thus, the operator $\mathcal{F}$ defined in (14) can be expressed in the form

$$
\mathcal{F}(x, z)(t)=\left(\mathcal{F}_{1}(x, z)(t), \mathcal{F}_{2}(x, z)(t)\right)=\mathcal{A}(x(t), z(t)) \mathcal{B}(x(t), z(t)),
$$

where $\mathcal{A}=\left(\mathcal{A}_{1}, \mathcal{A}_{2}\right), \mathcal{B}=\left(\mathcal{B}_{1}, \mathcal{B}_{2}\right)$ and $\mathcal{A}_{i}, \mathcal{B}_{i}: \overline{\mathcal{X}_{r}(0)} \rightarrow X$ for $i=1,2$ are defined by

$$
\begin{aligned}
\mathcal{A}_{1}(x(t), z(t))= & \mathcal{H}(t, x, z), \quad \mathcal{A}_{2}(x(t), z(t))=\mathcal{G}(t, x, z), \\
\mathcal{B}_{1}(x(t), z(t))= & -\int_{0}^{t} \frac{(t-s)^{\omega-1}}{\Gamma(\omega)} \mathcal{K}_{1}(s, x(s), z(s)) d s \\
& +\int_{0}^{1} \frac{(1-s)^{\omega-1}}{\Gamma(\omega)} \mathcal{K}_{1}(s, x(s), z(s)) d s \\
& +\frac{(t-1)}{\Delta_{1} \Gamma(\omega-\mu)} \int_{0}^{\delta_{1}}\left(\delta_{1}-s\right)^{\omega-\mu-1} \mathcal{K}_{1}(s, x(s), z(s)) d s, \\
\mathcal{B}_{2}(x(t), z(t))= & -\int_{0}^{t} \frac{(t-s)^{\epsilon-1}}{\Gamma(\epsilon)} \mathcal{K}_{2}(s, x(s), z(s)) d s \\
& +\int_{0}^{1} \frac{(1-s)^{\epsilon-1}}{\Gamma(\epsilon)} \mathcal{K}_{2}(s, x(s), z(s)) d s \\
& +\frac{(t-1)}{\Delta_{2} \Gamma(\epsilon-v)} \int_{0}^{\delta_{2}}\left(\delta_{2}-s\right)^{\epsilon-\nu-1} \mathcal{K}_{2}(s, x(s), z(s)) d s .
\end{aligned}
$$


Step $\mathrm{I}$. The operators $\mathcal{A}_{1}, \mathcal{A}_{2}$ are Lipschitz on $X$. For this, let $\left(x_{1}, z_{1}\right),\left(x_{2}, z_{2}\right) \in X \times X$ and $t \in[0,1]$; then by $\left(C_{1}\right)$ we get

$$
\begin{aligned}
\left|\mathcal{A}_{1}\left(x_{1}, z_{1}\right)(t)-\mathcal{A}_{1}\left(x_{2}, z_{2}\right)(t)\right| & =\left|\mathcal{H}\left(x_{1}, z_{1}\right)-\mathcal{H}\left(x_{2}, z_{2}\right)\right| \\
& \leq \mu_{1}\left|x_{1}(t)-z_{1}(t)\right|+\mu_{2}\left|x_{2}(t)-z_{2}(t)\right| \\
& \leq\left(\mu_{1}+\mu_{2}\right)\left\|\left(x_{1}, z_{1}\right)-\left(x_{2}, z_{2}\right)\right\| .
\end{aligned}
$$

Similarly, we obtain

$$
\begin{aligned}
\left|\mathcal{A}_{2}\left(x_{1}, z_{1}\right)(t)-\mathcal{A}_{2}\left(x_{2}, z_{2}\right)(t)\right| & =\left|\mathcal{G}\left(x_{1}, z_{1}\right)-\mathcal{G}\left(x_{2}, z_{2}\right)\right| \\
& \leq \mu_{3}\left|x_{1}(t)-z_{1}(t)\right|+\mu_{2}\left|x_{4}(t)-z_{2}(t)\right| \\
& \leq\left(\mu_{3}+\mu_{4}\right)\left\|\left(x_{1}, z_{1}\right)-\left(x_{2}, z_{2}\right)\right\| .
\end{aligned}
$$

From (25) and (26) we obtain

$$
\begin{aligned}
\left|\mathcal{A}\left(x_{1}, z_{1}\right)(t)-\mathcal{A}\left(x_{2}, z_{2}\right)(t)\right| & =\left|\left(\mathcal{A}_{1}, \mathcal{A}_{2}\right)\left(x_{1}, z_{1}\right)-\left(\mathcal{A}_{1}, \mathcal{A}_{2}\right)\left(x_{2}, z_{2}\right)\right| \\
& \leq\left(\mu_{1}+\mu_{2}+\mu_{3}+\mu_{4}\right)\left\|\left(x_{1}, z_{1}\right)-\left(x_{2}, z_{2}\right)\right\| .
\end{aligned}
$$

Thus, (27) implies that $\mathcal{A}$ is Lipschitz on $X \times X$ with Lipschitz constant $\mu_{1}+\mu_{2}+\mu_{3}+\mu_{4}$.

Step II. In this step, we show that the operator $\mathcal{B}$ is continuous. For this, let us assume that $\left\{x_{n}\right\},\left\{z_{n}\right\}$ are convergent sequences such that $x_{n} \rightarrow x, z_{n} \rightarrow z$ as $n \rightarrow+\infty$. From the continuity of $\mathcal{K}_{1}$ it follows that $\mathcal{K}_{1}\left(t, x_{n}(t), z_{n}(t)\right) \rightarrow \mathcal{K}_{1}(t, x(t), z(t)), \mathcal{K}_{2}\left(t, x_{n}(t), z_{n}(t)\right) \rightarrow$ $\mathcal{K}_{2}(t, x(t), z(t))$. By the Lebesgue dominated theorem, we have

$$
\begin{aligned}
\lim _{n \rightarrow \infty} \mathcal{B}_{1}\left(x_{n}, z_{n}\right)= & \lim _{n \rightarrow \infty}\left(-\int_{0}^{t} \frac{(t-s)^{\omega-1}}{\Gamma(\omega)} \mathcal{K}_{1}\left(s, x_{n}(s), z_{n}(s)\right) d s\right. \\
& +\int_{0}^{1} \frac{(1-s)^{\omega-1}}{\Gamma(\omega)} \mathcal{K}_{1}\left(s, x_{n}(s), z_{n}(s)\right) d s \\
& \left.+\frac{(t-1)}{\Delta_{1} \Gamma(\omega-\mu)} \int_{0}^{\delta_{1}}\left(\delta_{1}-s\right)^{\omega-\mu-1} \mathcal{K}_{1}\left(s, x_{n}(s), z_{n}(s)\right) d s\right) \\
= & -\int_{0}^{t} \frac{(t-s)^{\omega-1}}{\Gamma(\omega)} \lim _{n \rightarrow \infty} \mathcal{K}_{1}\left(s, x_{n}(s), z_{n}(s)\right) d s \\
& +\int_{0}^{1} \frac{(1-s)^{\omega-1}}{\Gamma(\omega)} \lim _{n \rightarrow \infty} \mathcal{K}_{1}\left(s, x_{n}(s), z_{n}(s)\right) d s \\
& +\frac{(t-1)}{\Delta_{1} \Gamma(\omega-\mu)} \int_{0}^{\delta_{1}}\left(\delta_{1}-s\right)^{\omega-\mu-1} \lim _{n \rightarrow \infty} \mathcal{K}_{1}\left(s, x_{n}(s), z_{n}(s)\right) d s \\
= & -\int_{0}^{t} \frac{(t-s)^{\omega-1}}{\Gamma(\omega)} \mathcal{K}_{1}(s, x(s), z(s)) d s \\
& +\int_{0}^{1} \frac{(1-s)^{\omega-1}}{\Gamma(\omega)} \mathcal{K}_{1}(s, x(s), z(s)) d s \\
& +\frac{(t-1)}{\Delta_{1} \Gamma(\omega-\mu)} \int_{0}^{\delta_{1}}\left(\delta_{1}-s\right)^{\omega-\mu-1} \mathcal{K}_{1}(s, x(s), z(s)) d s \\
= & \mathcal{B}_{1}(x, z) .
\end{aligned}
$$


Similarly, we obtain

$$
\begin{aligned}
\lim _{n \rightarrow 0} \mathcal{B}_{2}\left(x_{n}, z_{n}\right)= & \lim _{n \rightarrow \infty}\left(-\int_{0}^{t} \frac{(t-s)^{\epsilon-1}}{\Gamma(\epsilon)} \mathcal{K}_{2}\left(s, x_{n}(s), z_{n}(s)\right) d s\right. \\
& +\int_{0}^{1} \frac{(1-s)^{\epsilon-1}}{\Gamma(\epsilon)} \mathcal{K}_{2}\left(s, x_{n}(s), z_{n}(s)\right) d s \\
& \left.+\frac{(t-1)}{\Delta_{2} \Gamma(\epsilon-v)} \int_{0}^{\delta_{2}}\left(\delta_{2}-s\right)^{\epsilon-\nu-1} \mathcal{K}_{2}\left(s, x_{n}(s), z_{n}(s)\right) d s\right) \\
= & -\int_{0}^{t} \frac{(t-s)^{\epsilon-1}}{\Gamma(\epsilon)} \lim _{n \rightarrow \infty} \mathcal{K}_{2}\left(s, x_{n}(s), z_{n}(s)\right) d s \\
& +\int_{0}^{1} \frac{(1-s)^{\epsilon-1}}{\Gamma(\epsilon)} \lim _{n \rightarrow \infty} \mathcal{K}_{2}\left(s, x_{n}(s), z_{n}(s)\right) d s \\
& +\frac{(t-1)}{\Delta_{2} \Gamma(\epsilon-v)} \int_{0}^{\delta_{2}}\left(\delta_{2}-s\right)^{\epsilon-\nu-1} \lim _{n \rightarrow \infty} \mathcal{K}_{2}\left(s, x_{n}(s), z_{n}(s)\right) d s \\
= & -\int_{0}^{t} \frac{(t-s)^{\epsilon-1}}{\Gamma(\epsilon)} \mathcal{K}_{2}(s, x(s), z(s)) d s \\
& +\int_{0}^{1} \frac{(1-s)^{\epsilon-1}}{\Gamma(\epsilon)} \mathcal{K}_{2}(s, x(s), z(s)) d s \\
& +\frac{(t-1)}{\Delta_{2} \Gamma(\epsilon-v)} \int_{0}^{\delta_{2}}\left(\delta_{2}-s\right)^{\epsilon-\nu-1} \mathcal{K}_{2}(s, x(s), z(s)) d s \\
= & \mathcal{B}_{2}(x, z) .
\end{aligned}
$$

By the help of (28), (29) we proved that the operator $\mathcal{B}=\left(\mathcal{B}_{1}, \mathcal{B}_{2}\right)$ is continuous for all $t \in[0,1]$.

Step III. In this step, we show that the operator $\mathcal{B}=\left(\mathcal{B}_{1}, \mathcal{B}_{2}\right)$ is compact on $\overline{\mathcal{X}_{r}(0)}$.

$$
\begin{aligned}
\left|\mathcal{B}_{1}(x, z)(t)\right|= & -\int_{0}^{t} \frac{(t-s)^{\omega-1}}{\Gamma(\omega)} \mathcal{K}_{1}(s, x(s), z(s)) d s \\
& +\int_{0}^{1} \frac{(1-s)^{\omega-1}}{\Gamma(\omega)} \mathcal{K}_{1}(s, x(s), z(s)) d s \\
& +\frac{(t-1)}{\Delta_{1} \Gamma(\omega-\mu)} \int_{0}^{\delta_{1}}\left(\delta_{1}-s\right)^{\omega-\mu-1} \mathcal{K}_{1}(s, x(s), z(s)) d s \mid \\
\leq & \int_{0}^{t} \frac{(t-s)^{\omega-1}}{\Gamma(\omega)}\left|\mathcal{M}_{1}(s)\right| d s+\int_{0}^{1} \frac{(1-s)^{\omega-1}}{\Gamma(\omega)}\left|\mathcal{M}_{1}(s)\right| d s \\
& +\frac{(t-1)}{\Delta_{1} \Gamma(\omega-\mu)} \int_{0}^{\delta_{1}}\left(\delta_{1}-s\right)^{\omega-\mu-1}\left|\mathcal{M}_{1}(s)\right| d s \\
\leq & \left(\frac{1}{\Gamma(\omega+1)}+\frac{1}{\Gamma(\omega+1)}+\frac{2 \delta_{1}^{\omega-\mu}}{\Delta_{1} \Gamma(\omega-\mu+1)}\right)\left\|\mathcal{M}_{1}\right\| \\
= & \mathcal{N}_{1}\left\|\mathcal{M}_{1}\right\| .
\end{aligned}
$$

Taking supremum over $t \in[0,1]$, we have

$$
\left\|\mathcal{B}_{1}(x, z)\right\| \leq \mathcal{N}_{1}\left\|\mathcal{M}_{1}\right\| \quad \forall(x, z) \in \overline{\mathcal{X}_{r}(0)} \times \overline{\mathcal{X}_{r}(0)}
$$


Similarly, we obtain

$$
\begin{aligned}
\left|\mathcal{B}_{2}(x(t), z(t))\right|= & -\int_{0}^{t} \frac{(t-s)^{\epsilon-1}}{\Gamma(\epsilon)} \mathcal{K}_{2}(s, x(s), z(s)) d s+\int_{0}^{1} \frac{(1-s)^{\epsilon-1}}{\Gamma(\epsilon)} \mathcal{K}_{2}(s, x(s), z(s)) d s \\
& +\frac{(t-1)}{\Delta_{2} \Gamma(\epsilon-v)} \int_{0}^{\delta_{2}}\left(\delta_{2}-s\right)^{\epsilon-v-1} \mathcal{K}_{2}(s, x(s), z(s)) d s \mid \\
\leq & \int_{0}^{t} \frac{(t-s)^{\epsilon-1}}{\Gamma(\epsilon)}\left|\mathcal{M}_{2}(s)\right| d s+\int_{0}^{1} \frac{(1-s)^{\epsilon-1}}{\Gamma(\epsilon)}\left|\mathcal{M}_{2}(s)\right| d s \\
& +\frac{(t-1)}{\Delta_{2} \Gamma(\epsilon-v)} \int_{0}^{\delta_{2}}\left(\delta_{2}-s\right)^{\epsilon-v-1}\left|\mathcal{M}_{2}(s)\right| d s \\
\leq & \left(\frac{1}{\Gamma(\epsilon+1)}+\frac{1}{\Gamma(\epsilon+1)}+\frac{2 \delta_{2}^{\epsilon-v}}{\Delta_{1} \Gamma(\epsilon-v+1)}\right)\left\|\mathcal{M}_{2}\right\| \\
= & \mathcal{N}_{2}\left\|\mathcal{M}_{2}\right\| .
\end{aligned}
$$

Taking the supremum of (32) over $t \in[0,1]$, we get

$$
\left\|\mathcal{B}_{2}(x, z)\right\| \leq \mathcal{N}_{2}\left\|\mathcal{M}_{2}\right\| \quad \forall(x, z) \in \overline{\mathcal{X}_{r}(0)} \times \overline{\mathcal{X}_{r}(0)} .
$$

From (31) and (32) it follows that

$$
\|\mathcal{B}(x, z)\| \leq \mathcal{N}_{1}\left\|\mathcal{M}_{1}\right\|+\mathcal{N}_{2}\left\|\mathcal{M}_{2}\right\| \quad \forall(x, z) \in \overline{\mathcal{X}_{r}(0)} \times \overline{\mathcal{X}_{r}(0)}
$$

which implies that $\mathcal{B}\left(\overline{\mathcal{X}_{r}(0)} \times \overline{\mathcal{X}_{r}(0)}\right)$ is a uniformly bounded set in $X \times X$.

Now we prove that the operator $\mathcal{B}$ is equicontinuous. For this, let us assume $0 \leq t_{1} \leq$ $t_{2} \leq 1$, then we have

$$
\begin{aligned}
\left|\mathcal{B}_{1}(x, z)\left(t_{2}\right)-\mathcal{B}_{1}(x, z)\left(t_{1}\right)\right|= & \mid \int_{0}^{t_{2}} \frac{\left(t_{2}-s\right)^{\omega-1}}{\Gamma(\omega)} \mathcal{K}_{1}(s, x(s), z(s)) d s \\
& -\int_{0}^{t_{1}} \frac{\left(t_{1}-s\right)^{\omega-1}}{\Gamma(\omega)} \mathcal{K}_{1}(s, x(s), z(s)) d s \\
& +\frac{\left(t_{2}-t_{1}\right)}{\Delta_{1} \Gamma(\omega-\mu)} \int_{0}^{\delta_{1}}\left(\delta_{1}-s\right)^{\omega-\mu-1} \mathcal{K}_{1}(s, x(s), z(s)) d s \mid \\
\leq & \int_{0}^{t_{2}} \frac{\left(t_{2}-s\right)^{\omega-1}}{\Gamma(\omega)} \mathcal{M}_{1}(s) d s-\int_{0}^{t_{1}} \frac{\left(t_{1}-s\right)^{\omega-1}}{\Gamma(\omega)} \mathcal{M}_{1}(s) d s \\
& +\frac{\left(t_{2}-t_{1}\right)}{\Delta_{1} \Gamma(\omega-\mu)} \int_{0}^{\delta_{1}}\left(\delta_{1}-s\right)^{\omega-\mu-1} \mathcal{M}_{1}(s) d s \\
\leq & \left(\frac{t_{2}^{\omega}-t_{1}^{\omega}}{\Gamma(\omega+1)}+\frac{\left(t_{2}-t_{1}\right) \delta_{1}^{\omega-\nu}}{\Delta_{1} \Gamma(\omega-\mu+1)}\right)\left\|\mathcal{M}_{1}\right\| .
\end{aligned}
$$

Similarly, we have

$$
\begin{aligned}
\left|\mathcal{B}_{2}(x, z)\left(t_{1}\right)-\mathcal{B}_{2}(x, z)\left(t_{2}\right)\right|= & \mid \int_{0}^{t_{2}} \frac{\left(t_{2}-s\right)^{\epsilon-1}}{\Gamma(\epsilon)} \mathcal{K}_{2}(s, x(s), z(s)) d s \\
& -\int_{0}^{t_{1}} \frac{\left(t_{1}-s\right)^{\epsilon-1}}{\Gamma(\epsilon)} \mathcal{K}_{2}(s, x(s), z(s)) d s
\end{aligned}
$$




$$
\begin{aligned}
& +\frac{\left(t_{2}-t_{1}\right)}{\Delta_{2} \Gamma(\epsilon-v)} \int_{0}^{\delta_{2}}\left(\delta_{2}-s\right)^{\epsilon-\nu-1} \mathcal{K}_{2}(s, x(s), z(s)) d s \mid \\
\leq & \int_{0}^{t_{2}} \frac{\left(t_{2}-s\right)^{\epsilon-1}}{\Gamma(\epsilon)} \mathcal{M}_{2}(s) d s-\int_{0}^{t_{1}} \frac{\left(t_{1}-s\right)^{\epsilon-1}}{\Gamma(\epsilon)} \mathcal{M}_{2}(s) d s \\
& +\frac{\left(t_{2}-t_{1}\right)}{\Delta_{2} \Gamma(\epsilon-v)} \int_{0}^{\delta_{2}}\left(\delta_{2}-s\right)^{\epsilon-\nu-1} \mathcal{M}_{2}(s) d s \\
\leq & \left(\frac{t_{2}^{\epsilon}-t_{1}^{\epsilon}}{\Gamma(\epsilon+1)}+\frac{\left(t_{2}-t_{1}\right) \delta_{2}^{\epsilon-\nu}}{\Delta_{2} \Gamma(\epsilon-v+1)}\right)\left\|\mathcal{M}_{2}\right\| .
\end{aligned}
$$

From (34) and (35) it follows that

$$
\begin{aligned}
\left|\mathcal{B}(x, z)\left(t_{1}\right)-\mathcal{B}(x, z)\left(t_{2}\right)\right| \leq & {\left[\frac{t_{2}^{\omega}-t_{1}^{\omega}}{\Gamma(\omega+1)}+\frac{\left(t_{2}-t_{1}\right) \delta_{1}^{\omega-\nu}}{\Delta_{1} \Gamma(\omega-\mu+1)}\right]\left\|\mathcal{M}_{1}\right\| } \\
& +\left[\frac{t_{2}^{\epsilon}-t_{1}^{\epsilon}}{\Gamma(\epsilon+1)}+\frac{\left(t_{2}-t_{1}\right) \delta_{2}^{\epsilon-\nu}}{\Delta_{2} \Gamma(\epsilon-v+1)}\right]\left\|\mathcal{M}_{2}\right\|,
\end{aligned}
$$

which implies that $\mathcal{B}(x, z)\left(t_{1}\right)-\mathcal{B}(x, z)\left(t_{2}\right) \rightarrow 0$ as $t_{1} \rightarrow t_{2}$. This shows that the operator $\mathcal{B}$ is equicontinuous and, by the Arzela-Ascoli theorem, the operator $\mathcal{B}$ is compact.

Step IV. We have $\gamma \mathcal{B}_{\max }<1$, where $\mathcal{B}_{\max }=\left\|\mathcal{B}\left(\overline{\mathcal{X}_{r}(0)}\right)\right\|=\sup \left\{\|\mathcal{B}(x)\|: x \in \overline{\mathcal{X}_{r}(0)}\right\}$, and $\mathcal{B}_{i, \text { max }}=\left\|\mathcal{B}_{i}\left(\overline{\mathcal{X}_{r}(0)}\right)\right\|=\sup \left\{\left\|\mathcal{B}_{i}(x): x \in \overline{\mathcal{X}_{r}(0)}\right\|\right\}$ for $i=1$, 2. Using (33) and hypothesis $\left(C_{3}\right)$, we have

$$
\mathcal{B}_{\max } \leq \gamma\left(\mathcal{N}_{1}\left\|\mathcal{M}_{1}\right\|+\mathcal{N}_{2}\left\|\mathcal{M}_{2}\right\|\right)<1 \quad \forall(x, z) \in \overline{\mathcal{X}_{r}(0)} \times \overline{\mathcal{X}_{r}(0)}
$$

In this step, we prove that the operator $\mathcal{F}$ maps bounded subset of $\mathcal{Y} \times \mathcal{Y}$ into a bounded set. Choose $r>0$ and define a bounded subset $\mathcal{S}$ of $\mathcal{Y} \times \mathcal{Y}$ by $\mathcal{S}=\{(x, z) \in \mathcal{Y} \times \mathcal{Y}$ : $\|(x, z)\| \leq r\}$. Now, for $(x, z) \in \mathcal{S}$ and $t \in[0,1], \lambda \in(0,1)$ such that $\|(x, z)\|=r$ and $(x, z)=$ $\lambda \mathcal{A}(x, z) \mathcal{B}(x, z)$. Using $\left(\mathrm{C}_{2}\right)$ and $\left(\mathrm{C}_{4}\right)$, we proceed

$$
\begin{aligned}
|x(t)|= & \lambda|\mathcal{H}(t, x, z)| \mid\left(-\int_{0}^{t} \frac{(t-s)^{\omega-1}}{\Gamma(\omega)} \mathcal{K}_{1}(s, x(s), z(s)) d s\right. \\
& +\int_{0}^{1} \frac{(1-s)^{\omega-1}}{\Gamma(\omega)} \mathcal{K}_{1}(s, x(s), z(s)) d s \\
& \left.+\frac{(t-1)}{\Delta_{1} \Gamma(\omega-\mu)} \int_{0}^{\delta_{1}}\left(\delta_{1}-s\right)^{\omega-\mu-1} \mathcal{K}_{1}(s, x(s), z(s)) d s\right) \mid \\
\leq & \lambda|\mathcal{H}(t, x, z)-\mathcal{H}(t, 0,0)+\mathcal{H}(t, 0,0)|\left(-\int_{0}^{t} \frac{(t-s)^{\omega-1}}{\Gamma(\omega)} \mathcal{M}_{1}(s) d s\right. \\
& \left.+\int_{0}^{1} \frac{(1-s)^{\omega-1}}{\Gamma(\omega)} \mathcal{M}_{1}(s) d s+(t-1) \int_{0}^{\delta_{1}} \frac{\left(\delta_{1}-s\right)^{\omega-\mu-1} \mathcal{M}_{1}(s) d s}{\Delta_{1} \Gamma(\omega-\mu)}\right) \mid \\
\leq & \lambda\left(\mu_{1}|x(t)|+\mu_{2}|z(t)|+\mathcal{H}_{0}\right)\left(\frac{2}{\Gamma(\omega+1)}+\frac{2 \delta_{1}^{\omega-\mu}}{\Delta_{1} \Gamma(\omega-\mu+1)}\right)\left\|\mathcal{M}_{1}\right\| \\
= & \lambda_{1}\left(\mu_{1}|x(t)|+\mu_{2}|z(t)|+\mathcal{H}_{0}\right) \mathcal{N}_{1}\left\|\mathcal{M}_{1}\right\| .
\end{aligned}
$$

Taking supremum over $t \in[0,1]$ and using $\left(\mathrm{C}_{4}\right)$, we obtain

$$
\|x\| \leq \lambda_{1}\left(\mu_{1}\|x\|+\mu_{2}\|z\|+\mathcal{H}_{0}\right) \mathcal{N}_{1}\left\|\mathcal{M}_{1}\right\|
$$


which implies that

$$
\|x\| \leq \lambda \frac{\left(\mu_{2}\|z\|+\mathcal{H}_{0}\right)}{1-\lambda \mu_{1} \mathcal{N}_{1}\left\|\mathcal{M}_{1}\right\|} \mathcal{N}_{1}\left\|\mathcal{M}_{1}\right\|
$$

Similarly, we have

$$
\|z\| \leq \lambda \frac{\left(\mu_{1}\|x\|+\mathcal{G}_{0}\right)}{1-\lambda \mu_{2} \mathcal{N}_{2}\left\|\mathcal{M}_{2}\right\|} \mathcal{N}_{2}\left\|\mathcal{M}_{2}\right\|
$$

By the help of (40) and (41), we get

$$
\begin{aligned}
\|(x, z)\|= & \|x\|+\|z\| \leq \lambda \frac{\left(\mu_{2}\|z\|+\mathcal{H}_{0}\right)}{1-\lambda \mu_{1} \mathcal{N}_{1}\left\|\mathcal{M}_{1}\right\|} \mathcal{N}_{1}\left\|\mathcal{M}_{1}\right\| \\
& +\lambda \frac{\left(\mu_{1}\|x\|+\mathcal{G}_{0}\right)}{1-\lambda \mu_{2} \mathcal{N}_{2}\left\|\mathcal{M}_{2}\right\|} \mathcal{N}_{2}\left\|\mathcal{M}_{2}\right\| \\
= & \lambda \frac{\mu_{2}\|z(t)\|}{1-\lambda \mathcal{N}_{1}\left\|\mathcal{M}_{1}\right\|} \mathcal{N}_{1}\left\|\mathcal{M}_{1}\right\|+\lambda \frac{\mathcal{H}_{0}}{1-\lambda \mu_{1} \mathcal{N}_{1}\left\|\mathcal{M}_{1}\right\|} \mathcal{N}_{1}\left\|\mathcal{M}_{1}\right\| \\
& +\lambda \frac{\mu_{1}\|x\|}{1-\lambda \mu_{2} \mathcal{N}_{2}\left\|\mathcal{M}_{2}\right\|} \mathcal{N}_{2}\left\|\mathcal{M}_{2}\right\|+\lambda \frac{\mathcal{G}_{0}}{1-\lambda \mu_{2} \mathcal{N}_{2}\left\|\mathcal{M}_{2}\right\|} \mathcal{N}_{2}\left\|\mathcal{M}_{2}\right\| .
\end{aligned}
$$

From (42) we obtain

$$
\|(x, z)\| \leq \frac{1}{1-\rho}\left(\frac{\lambda \mathcal{H}_{0} \mathcal{N}_{1}\left\|\mathcal{M}_{1}\right\|}{1-\lambda \mu_{1} \mathcal{N}_{1}\left\|\mathcal{M}_{1}\right\|}+\frac{\lambda \mathcal{G}_{0} \mathcal{N}_{2}\left\|\mathcal{M}_{2}\right\|}{1-\mu_{2} \lambda \mathcal{N}_{2}\left\|\mathcal{M}_{2}\right\|}\right)<r
$$

which contradicts our supposition $\|(x, z)\|=r$. Thus, the operator $(x, z)=\mathcal{A}(x, z) \mathcal{B}(x, z)$ has a solution in $\overline{\mathcal{X}_{r}(0)} \times \overline{\mathcal{X}_{r}(0)}$ which is a solution of system (1). This completes the proof of the theorem.

Example 11 Consider the following system of fractional order hybrid differential equations:

$$
\begin{aligned}
& \mathcal{D}^{2.5}\left(\frac{x(t)}{100+\frac{(|x(t)|+|z(t)|) \sin (t)}{50}}\right)=\frac{1+x(t) \sin (t)}{1+|x(t)|+|z(t)|}, \\
& \mathcal{D}^{2.5}\left(\frac{z(t)}{100+\frac{(|x(t)|+|z(t)|) \cos (t)}{50}}\right)=\frac{1+x(t) \cos (t)}{1+|x(t)|+|z(t)|},
\end{aligned}
$$

with initial and boundary conditions as defined in (1) for $\delta_{1}=\delta_{2}=\mu=\nu=0.5$ and $t \in$ $[0,1]$. Then system (44) satisfies all the conditions of Theorem 10. From (44) we have $\mathcal{H}(t, x, z)=100+\frac{(|x(t)|+|z(t)|) \sin (t)}{50}, \mathcal{G}(t, x, z)=100+\frac{(|x(t)|+|z(t)|) \cos (t)}{50} .\left|\mathcal{H}\left(t, x_{2}, z_{1}\right)-\mathcal{H}\left(t, x_{1}, z_{1}\right)\right| \leq$ $\frac{1}{50}\left(\left\|x_{2}-x_{1}\right\|+\left\|z_{2}-z_{1}\right\|\right),\left|\mathcal{G}\left(t, x_{2}, z_{1}\right)-\mathcal{G}\left(t, x_{1}, z_{1}\right)\right| \leq \frac{1}{50}\left(\left\|x_{2}-x_{1}\right\|+\left\|z_{2}-z_{1}\right\|\right)$, which implies $\mu_{i}=\frac{1}{50}$ for $i=1,2,3,4$. By using these values, we deduce $\beta \geq 67.7022$, we choose $\beta=70$. Thus system (44) has a solution in $\overline{\mathcal{X}_{70}(0)} \times \overline{\mathcal{X}_{70}(0)}$.

\subsection{Uniqueness of solutions}

In this subsection, we provide uniqueness of solutions for CSHFDEs (1). For this, we use BCP. 
Theorem 12 Assume that $\left(C_{1}\right)$ and $\left(C_{2}\right)$ hold. If $\mu\left(\mathcal{N}_{1}+\mathcal{N}_{2}\right)\left(\left\|\mathcal{M}_{1}\right\|+\left\|\mathcal{M}_{2}\right\|\right)<1$, then CSHFDEs (1) has a unique solution.

Proof Define $\mathcal{H}_{0}=\sup _{t \in[0,1]}|\mathcal{H}(t, 0,0)|, \mathcal{G}_{0}=\sup _{t \in[0,1]}|\mathcal{G}(t, 0,0)|$ and $\mu=\mu_{1}+\mu_{2}+\mu_{3}+\mu_{4}$. Choose $\beta \geq \frac{\mu\left(\mathcal{H}_{0}+\mathcal{G}_{0}\right)\left(\mathcal{N}_{1}+\mathcal{N}_{2}\right)\left(\left\|\mathcal{M}_{1}\right\|+\left\|\mathcal{M}_{2}\right\|\right)}{1-\mu\left(\mathcal{N}_{1}+\mathcal{N}_{2}\right)\left(\left\|\mathcal{M}_{1}\right\|+\left\|\mathcal{M}_{2}\right\|\right)}$, where $\mathcal{N}_{1}, \mathcal{N}_{2}$ are defined in (17), (18), respectively. Define a set $\bar{S}_{\beta}=\{(x, z)(t) \in X \times X:\|(x, z)\| \leq \beta\}$. Then, for $(x, z) \in \bar{S}_{\beta}$, we have

$$
\begin{aligned}
&\left|\mathcal{F}_{1}(x(t), z(t))\right||\mathcal{H}(t, x, z)| \mid\left(-\int_{0}^{t} \frac{(t-s)^{\omega-1}}{\Gamma(\omega)} \mathcal{K}_{1}(s, x(s), z(s)) d s\right. \\
& \quad+\int_{0}^{1} \frac{(1-s)^{\omega-1}}{\Gamma(\omega)} \mathcal{K}_{1}(s, x(s), z(s)) d s \\
&\left.\quad+\frac{(t-1)}{\Delta_{1} \Gamma(\omega-\mu)} \int_{0}^{\delta_{1}}\left(\delta_{1}-s\right)^{\omega-\mu-1} \mathcal{K}_{1}(s, x(s), z(s)) d s\right) \mid \\
& \leq|\mathcal{H}(t, x, z)-\mathcal{H}(t, 0,0)+\mathcal{H}(t, 0,0)|\left(\int_{0}^{t} \frac{(t-s)^{\omega-1}}{\Gamma(\omega)}\left\|\mathcal{M}_{1}(s)\right\| d s\right. \\
&\left.\quad+\int_{0}^{1} \frac{(1-s)^{\omega-1}}{\Gamma(\omega)}\left\|\mathcal{M}_{1}(s)\right\| d s+\frac{(t-1)}{\Delta_{1} \Gamma(\omega-\mu)} \int_{0}^{\delta_{1}}\left(\delta_{1}-s\right)^{\omega-\mu-1}\left\|\mathcal{M}_{1}(s)\right\| d s\right) \\
& \leq\left(\mu_{1}|x(t)|+\mu_{2}|z(t)|+\mathcal{H}_{0}\right)\left(\frac{2}{\Gamma(\omega+1)}+\frac{2 \delta_{1}^{\omega-\mu}}{\Delta_{1} \Gamma(\omega-\mu+1)}\right)\left\|\mathcal{M}_{1}\right\| \\
&=\left(\mu_{1}|x(t)|+\mu_{2}|z(t)|+\mathcal{H}_{0}\right) \mathcal{N}_{1}\left\|\mathcal{M}_{1}\right\| .
\end{aligned}
$$

Taking supremum over $t \in[0,1]$, we obtain

$$
\begin{aligned}
\left\|\mathcal{F}_{1}(x, z)\right\| & \leq\left(\mu_{1}+\mu_{2}\right)\left(\|x\|+\|z\|+\mathcal{H}_{0}\right) \mathcal{N}_{1}\left\|\mathcal{M}_{1}\right\| \\
& \leq\left(\mu_{1}+\mu_{2}\right)\left(\beta+\mathcal{H}_{0}\right) \mathcal{N}_{1}\left\|\mathcal{M}_{1}\right\| .
\end{aligned}
$$

Similarly, we have

$$
\begin{aligned}
\left\|\mathcal{F}_{2}(x, z)\right\| & \leq\left(\mu_{3}+\mu_{4}\right)\left(\|x\|+\|z\|+\mathcal{G}_{0}\right) \mathcal{N}_{2}\left\|\mathcal{M}_{2}\right\| \\
& \leq\left(\mu_{3}+\mu_{4}\right)\left(\beta+\mathcal{G}_{0}\right) \mathcal{N}_{2}\left\|\mathcal{M}_{2}\right\| .
\end{aligned}
$$

By the help of (46) and (47), we get

$$
\begin{aligned}
\|\mathcal{F}(x, z)\| \leq & \left(\mu_{1}+\mu_{2}\right)\left(\beta+\mathcal{H}_{0}\right) \mathcal{N}_{1}\left\|\mathcal{M}_{1}\right\| \\
& +\left(\mu_{3}+\mu_{4}\right)\left(\beta+\mathcal{G}_{0}\right) \mathcal{N}_{2}\left\|\mathcal{M}_{2}\right\| \\
\leq & \left(\mu_{1}+\mu_{2}+\mu_{3}+\mu_{4}\right)\left(\beta+\mathcal{H}_{0}+\mathcal{G}_{0}\right)\left(\mathcal{N}_{1}+\mathcal{N}_{2}\right)\left(\left\|\mathcal{M}_{1}\right\|+\left\|\mathcal{M}_{2}\right\|\right) \leq \beta .
\end{aligned}
$$

Now, for $\left(x_{1}, z_{1}\right)(t),\left(x_{2}, z_{2}\right)(t) \in X \times X$ and any $t \in[0,1]$, we have

$$
\begin{aligned}
& \left|\mathcal{F}_{1}\left(x_{2}, z_{2}\right)(t)-\mathcal{F}_{1}\left(x_{1}, z_{1}\right)(t)\right| \\
& \quad \leq\left|\mathcal{H}\left(t, x_{2}, z_{2}\right)-\mathcal{H}\left(t, x_{1}, z_{1}\right)\right|\left\{\int_{0}^{t} \frac{(t-s)^{\omega-1}}{\Gamma(\omega)}\left|\mathcal{M}_{1}(s)\right| d s\right.
\end{aligned}
$$




$$
\begin{aligned}
& \left.+\int_{0}^{1} \frac{(1-s)^{\omega-1}}{\Gamma(\omega)}\left|\mathcal{M}_{1}(s)\right| d s+|t-1| \int_{0}^{\delta_{1}} \frac{\left(\delta_{1}-s\right)^{\omega-\mu-1}\left|\mathcal{M}_{1}(s)\right| d s}{\Delta_{1} \Gamma(\omega-\mu)}\right\} \\
\leq & \left(\mu_{1}\left|x_{2}-x_{1}\right|+\mu_{2}\left|z_{2}-z_{1}\right|\right)\left(\frac{2}{\Gamma(\omega+1)}+\frac{2 \delta_{1}^{\omega-\mu}}{\Delta_{1} \Gamma(\omega-\mu+1)}\right)|\mathcal{M}(t)| \\
\leq & \left(\mu_{1}+\mu_{2}\right) \mathcal{N}_{1}\left|\mathcal{M}_{1}(t)\right|\left(\left|x_{2}-x_{1}\right|+\left|z_{2}-z_{1}\right|\right) .
\end{aligned}
$$

Taking the supremum over $t \in[0,1]$, we obtain

$$
\begin{aligned}
& \left\|\mathcal{F}_{1}\left(x_{2}, z_{2}\right)-\mathcal{F}_{1}\left(x_{1}, z_{1}\right)\right\| \\
& \quad \leq\left(\mu_{1}+\mu_{2}\right) \mathcal{N}_{1}\left\|\mathcal{M}_{1}\right\|\left(\left\|x_{2}-x_{1}\right\|+\left\|z_{2}-z_{1}\right\|\right) .
\end{aligned}
$$

Similarly, we obtain

$$
\begin{aligned}
& \left\|\mathcal{F}_{2}\left(x_{2}, z_{2}\right)-\mathcal{F}_{2}\left(x_{1}, z_{1}\right)\right\| \\
& \quad \leq\left(\mu_{3}+\mu_{4}\right) \mathcal{N}_{2}\left\|\mathcal{M}_{2}\right\|\left(\left\|x_{2}-x_{1}\right\|+\left\|z_{2}-z_{1}\right\|\right) .
\end{aligned}
$$

From (50) and (51) it follows that

$$
\begin{aligned}
& \left\|\mathcal{F}\left(x_{2}, z_{2}\right)-\mathcal{F}\left(x_{1}, z_{1}\right)\right\| \\
& \quad \leq \mu\left(\mathcal{N}_{1}+\mathcal{N}_{2}\right)\left(\left\|\mathcal{M}_{1}\right\|+\left\|\mathcal{M}_{2}\right\|\right)\left(\left\|x_{2}-x_{1}\right\|+\left\|z_{2}-z_{1}\right\|\right),
\end{aligned}
$$

which implies that $\mathcal{F}$ is a contraction. By the help of Banach's contraction principle the operator $\mathcal{F}$ has a unique fixed point which is the unique solution of CSHFDEs (1). This completes the proof.

Example 13 Consider the following system of fractional order hybrid differential equations:

$$
\begin{aligned}
& \mathcal{D}^{2.5}\left(\frac{x(t)}{10+\frac{(|x(t)|+|z(t)|) \sin (t)}{50}}\right)=\frac{1+x(t) \sin (t)}{2+|x(t)|+|z(t)|}, \\
& \mathcal{D}^{2.5}\left(\frac{z(t)}{10+\frac{(|x(t)|+|z(t)| \mid \cos (t)}{50}}\right)=\frac{1+x(t) \cos (t)}{2+|x(t)|+|z(t)|},
\end{aligned}
$$

with initial and boundary conditions as defined in (1) for $\delta_{1}=\delta_{2}=\mu=v=0.5$. From system (44) we have $\mathcal{H}(t, x, z)=10+\frac{(|x(t)|+|z(t)|) \sin (t)}{50}, \mathcal{G}(t, x, z)=10+\frac{(|x(t)|+|z(t)|) \cos (t)}{50} . \mid \mathcal{H}\left(t, x_{2}, z_{1}\right)-$ $\mathcal{H}\left(t, x_{1}, z_{1}\right)\left|\leq \frac{1}{50}\left(\left\|x_{2}-x_{1}\right\|+\left\|z_{2}-z_{1}\right\|\right),\right| \mathcal{G}\left(t, x_{2}, z_{1}\right)-\mathcal{G}\left(t, x_{1}, z_{1}\right) \mid \leq \frac{1}{50}\left(\left\|x_{2}-x_{1}\right\|+\left\|z_{2}-z_{1}\right\|\right)$, which implies $\mu_{i}=\frac{1}{50}$ for $i=1,2,3$, 4. By using these values, we deduce $\beta \geq 6.77022$, we choose $\beta=7 . \mu\left(\mathcal{N}_{1}+\mathcal{N}_{2}\right)\left(\left\|\mathcal{M}_{1}\right\|+\left\|\mathcal{M}_{2}\right\|\right) \leq 0.252917<1$. Therefore, system (53) satisfies the assumptions of Theorem 12. Thus, system (53) has a unique solution in $\overline{\mathcal{X}_{7}(0)} \times \overline{\mathcal{X}_{7}(0)}$.

\section{Competing interests}

The authors declare that they have no competing interests. 


\section{Author details}

${ }^{1}$ Department of Mathematics and Computer Science, Cankaya University, Ankara, 06530, Turkey. ${ }^{2}$ Institute of Space Sciences, P.O. Box MG-23, Magurele-Bucharest, 76900, Romania. ${ }^{3}$ Department of Mathematics, University of Malakand, Dir Lower, P.O. Box 18000, Chakdara, Khybarpukhtunkhwa, Pakistan. ${ }^{4}$ Shaheed Benazir Bhutto University, Dir Upper, P.O. Box 18000, Sharingal, Khybarpukhtunkhwa, Pakistan. ${ }^{5}$ Department of Mathematical Sciences, University of South Africa (UNISA), P.O. Box 392, Pretoria, 0003, South Africa. ${ }^{6}$ Department of Mathematics, University of Mazandaran, P.O. Box 47416-95447, Babolsar, Iran. ${ }^{7}$ Department of Mathematics, Faculty of Basic Science, Babol University of Technology, P.O. Box 47148-71167, Babol, Iran.

\section{Acknowledgements}

We are thankful to the referees and editor for their valuable comments which improved the quality of the paper.

Received: 14 January 2015 Accepted: 1 October 2015 Published online: 15 October 2015

\section{References}

1. Hilfer, R (ed.): Application of Fractional Calculus in Physics. World Scientific, Singapore (2000)

2. Kilbas, AA, Srivastava, HM, Trujillo, JJ: Theory and Applications of Fractional Differential Equations. North-Holland Mathematics Studies, vol. 24. North-Holland, Amsterdam (2006)

3. Anastassiou, GA: On right fractional calculus. Chaos Solitons Fractals 42(1), 365-376 (2009)

4. Houas, M, Dahmani, Z: New results for a coupled system of fractional differential equations. Facta Univ., Ser. Math. Inform. 28(2), 133-150 (2013)

5. Khan, RA, Khan, A, Samad, A, Khan, H: On existence of solutions for fractional differential equations with $P$-Laplacian operator. J. Fract. Calc. Appl. 5(2), 28-37 (2014)

6. Rehman, M, Khan, RA: Positive solutions to coupled system of fractional differential equations. Int. J. Nonlinear Sci. 10(1), 96-104 (2010)

7. Li, M, Liu, Y: Existence and uniqueness of positive solutions for a coupled system of nonlinear fractional differential equations. Open J. Appl. Sci. 3, 53-61 (2013). doi:10.4236/ojapps.2013.31B1011

8. Ntouyas, SK, Obaid, M: A coupled system of fractional differential equations with nonlocal integral boundary conditions. Adv. Differ. Equ. 2012, Article ID 130 (2012)

9. Hedrih, KR: Fractional order hybrid system dynamics. Proc. Appl. Math. Mech. 13, 25-26 (2013)

10. Dhage, BC: Quadratic perturbations of periodic boundary value problems of second order ordinary differential equations. Differ. Equ. Appl. 2, 465-486 (2010)

11. Dhage, BC, Lakshmikantham, V: Basic results on hybrid differential equations. Nonlinear Anal. Hybrid Syst. 4, 414-424 (2010)

12. Ahmad, B, Ntouyas, SK, Alsaedi, A: Existence results for a system of coupled hybrid fractional differential equations. Sci. World J. 2014, Article ID 426438 (2014)

13. Herzallah, MAE, Baleanu, D: On fractional order hybrid differential equations. Abstr. Appl. Anal. 2014, Article ID 389386 (2014)

14. Ahmad, B, Ntouyas, SK: Initial value problems for hybrid Hadamard fractional differential equations. Electron. J. Differ. Equ. 2014, 161 (2014)

15. Dhage, BC: On a fixed point theorem in Banach algebras with applications. Appl. Math. Lett. 18, 273-280 (2005)

\section{Submit your manuscript to a SpringerOpen ${ }^{\circ}$ journal and benefit from:}

- Convenient online submission

Rigorous peer review

- Immediate publication on acceptance

Open access: articles freely available online

- High visibility within the field

- Retaining the copyright to your article 